\title{
Customer Review-driven Function Formulation for Design Education
}

\section{Dr. Ang Liu, University of New South Wales}

Dr. Ang Liu is a senior lecturer at the School of Mechanical and Manufacturing Engineering, University of New South Wales, Australia

\section{Mr. Yuchen Wang, University of New South Wales}

Yuchen Wang is an undergraduate Aerospace Engineering student in University of New South Wales,Australia. He assists in engineering design education, meanwhile, his student off-class research focuses on space theme projects such as sun sensor and thermal controller.

\section{Dr. Yun Dai, University of Southern California}

Dr. Yun Dai is a postdoc research fellow in the Viterbi School of Engineering, at the University of Southern California, and also the program manager of the Viterbi iPodia Program (ViP). Her research interest broadly involves engineering education, learning technology, and intercultural education. 


\section{Customer Review driven Function Formulation for Design Education}

Product design is rapidly shifting from the conventional paradigm of creativity-oriented and model-based design toward a new paradigm of user-centered and data-driven design. Against such a background, this paper discusses the values of massively crowdsourced customer reviews for engineering design education. Recently, customers become growingly motivated to share their first-hand experience of using a product on various ecommerce and social platforms in the format of customer reviews (CRs). In practice, these informative CRs are increasingly employed by professional designers to support their early-stage design decision making. Unlike expert designers who are more comfortable at comprehending ill-structured CRs without any predefined hypothesis or structured design process, student designers should be carefully guided to uncover the implicit values of CRs for their design projects. Based on a novel integration of relevant design methods and recommendation approaches, this paper presents a CR-driven function formulation method. Student designers can follow the new method to abstract useful information from readily available customer reviews to formulate a unique engineering design problem as a set of functional requirements.

\section{Introduction}

Product design is rapidly shifting from the conventional paradigm of creativity-oriented and model-based design toward a new paradigm of user-centered and data-driven design. As a result of the information explosion, the information that is directly or indirectly useful for product design has increased in an exponential manner [1-2]. Among the rich information that is readily available on the Internet, designers are especially interested in the information that is purposefully created, published, and shared by customers. Customers become growingly motivated to share their first-hand experiences of using a product on various Internet platforms (e.g., ecommerce platform, social networking platform, and review platform) in the form of customer reviews (CRs). Take a popular product "Kindle E-reader" for instance, by the time this study is conducted, there are over 37,000 customer reviews about this product that are published on Amazon.com. In design practice, these readily available CRs are often leveraged by professional designers to support their early-stage design decision-making. To date, few efforts have been devoted to making the value of CR's visible to student designers in the context of input to their design projects

Unlike expert designers who are more comfortable at comprehending ill-structured CRs without any predefined hypothesis or structured design process, student designers should be carefully guided to uncover values of CRs for their design projects. Therefore, a structured design method should be prescribed for student designers to make full usage of CRs. Compared to concept generation that is well-supported by many existing design methods [35], a more challenging task for student designers is function formulation (i.e., how to formulate a set of explicit functional requirements for the to-be-designed artifact) [6]. In practice, new functions are primarily formulated by senior designers based on their extrapolation of customer voices solicited through lead user engagement [7], brainstorming [8], focus group, individual interview, survey, ethnography [9], etc. In the context of engineering education, however, it is challenging for student designers to directly apply these methods in their design projects, largely due to the lack of access to the "real" customers and their "true" voices. This challenge is especially true since many of the design projects are characterized by an open-ended and ill-defined problem statement. 
This paper presents a customer review driven function formulation (CR-FF) method, which guides student designers to leverage the massively crowdsourced customer reviews (CR) to support the project-based learning in engineering design classes.

\section{Literature Review of Relevant Work}

Function plays a critical role in transforming intangible customer needs to more tangible design parameter under various design constraints [10]. New functions are formulated by designers and assigned to an artifact. On the one hand, a set of functions outlines a concrete target and criteria for the following design activities such as concept generation, evaluation, selection, improvement, and optimization. On the other hand, function formulation is one of the most challenging design activities, especially for novice designers. This is determined by the nature of functions as being intangible, solution-neutral, and dynamic. In design education practice, it is by no means uncommon that student designers often fail to clearly distinguish function from customer need, product behavior, and design constraint [11].

Function can be represented as a relationship between input and output of energy, material, and signal [12-13]. According to the Function-Behaviour-Structure (FBS) framework [14], function is defined as the purpose of an artifact (i.e., what the artifact is intended to do). Function plays a critical role in bridging the problem space and the solution space. Based on the Axiomatic Design Theory [10], functional domain is one of the four fundamental design domains, and functional requirements link customer needs to design parameters. Based on the FBS framework, the operation of "formulation" is repeatedly performed firstly to transform external requirement to function, next to map function to expected behavior. In addition, there are many previous studies about function representation and organization [15-17].

Customer reviews (CR) can be crowdsourced from end users. Crowdsourcing means the practice of seeking for small contributions from a large number of end users to collectively accomplish a large-scale task [18]. Strictly speaking, crowdsourcing is not a completely new approach. For example, manufacturers have been following the crowdsourcing model for a long time to solicit new ideas from end users through various design competitions such as the LEGO Ideas Competition and Toyota Dream Car Art Contest. Prior to the Internet era, nevertheless, it was logistically difficult to aggregate small contributions from a massive number of end users. In light of the growing popularity of mobile devices, mobile crowdsourcing is becoming increasingly accepted [19]. The crowdsourcing model is expanded towards crowd-funding, crowd-searching, crowd-solving [20], etc. To date, few efforts have been devoted to developing the model of crowd-learning (i.e., the practice of soliciting small contributions from many learners to jointly accomplish a common learning task), apart from some notable exceptions. For example, crowdsourcing techniques are being used to assembly small contributions of separate learning contents into integrated content [21].

It is fairly common that student designers often conduct self-made surveys to collect information from peer classmates or schoolmates, which can be regarded as a special form of crowd-learning. Many online tools (e.g., instant polling and survey services) are available for students to conduct crowd-learning on social media/networking platforms and learning management system. For design education, crowd-learning is especially applicable for those design activities that depend on divergent thinking to perform, for instance, problem formulation and idea generation. For example, Figure 1 illustrates a word-cloud of "stakeholders who are relevant to the design of a computer input device", which was created 
by crowdsourcing inputs from student designers based on a second-year design course (i.e., MMAN2100 Engineering Design II) taught by the authors at University of New South Wales.

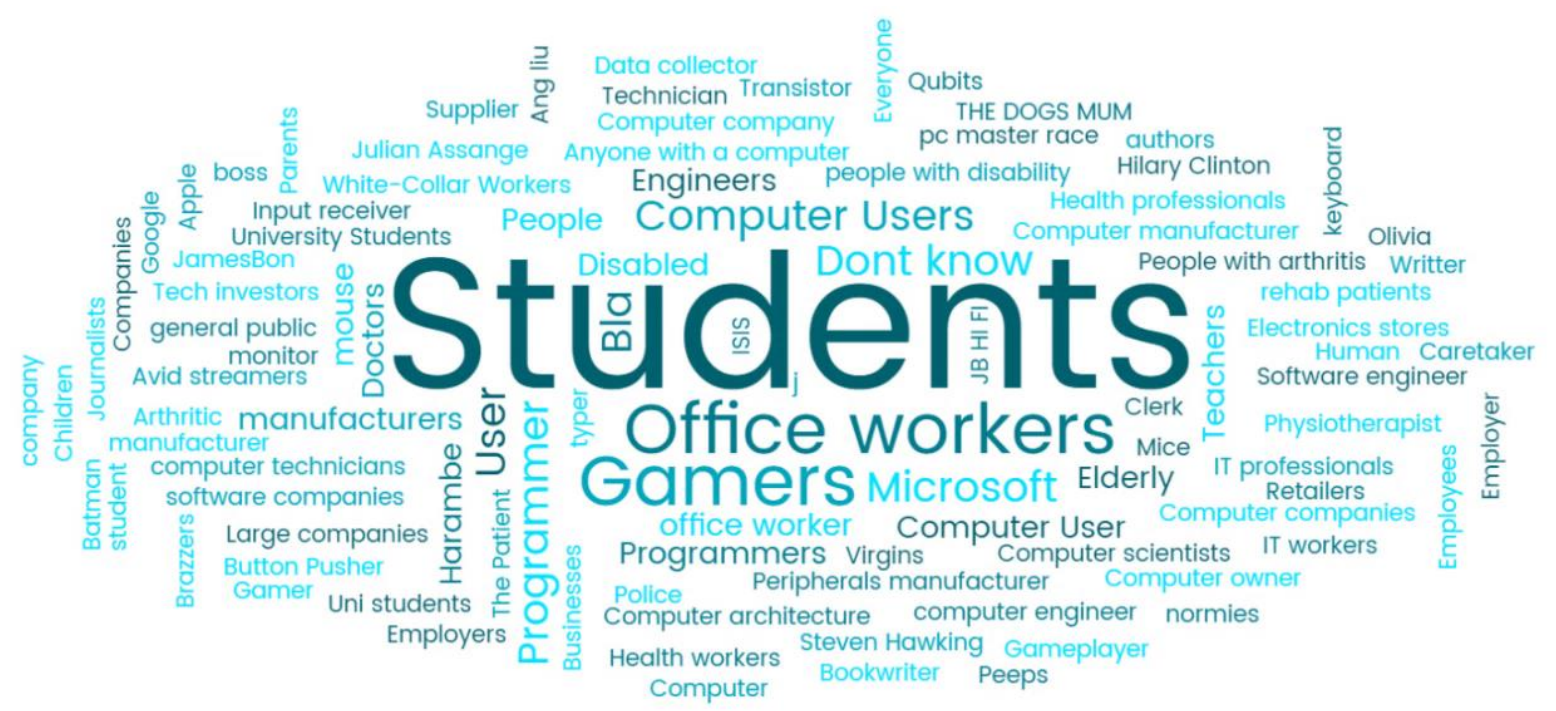

Figure 1. Illustration of word-cloud crowdsourced project

A more viable strategy than the direct crowd-learning model is to guide student designers to abstract useful information from those massively crowdsourced customer reviews (CR) that are readily available on the Internet. Traditionally, the values of CRs are mostly explored from the business perspective. On the one hand, CRs can increase customer attachment to an ecommerce platform through the sense of "community", and hence enable retailers to make personalized product recommendations [22]. On the other hand, CRs empower customers to make their voices heard by a broader audience and hence enable the fellow customers to make more informed purchase decisions [23]. The values of CRs are increasingly recognized by professional designers, who often follow the netnography method to analyze consumer opinions in online communities [24].

CRs can be collected from a variety of online channels such as ecommerce platforms, search engines, social media/networking platforms, video sharing website, photo sharing websites, product forums, and so forth. The CRs used in this study were all collected from the ecommerce platform of Amazon.com. Since CRs ubiquitously exist in different Internet platforms, some advanced mining techniques can be employed to aggregate CRs about the same product [25]. Rich information can be abstracted from CRs. Take a typical CR published on Amazon.com for example, the information relevant to design include product rating, reviewer ranking, textual content, photo and video, comments and Q\&A, and peer evaluation of a CR's helpfulness. The massively crowdsourced customer reviews should be systemically filtered and analyzed to be useful for design practice and education. This is necessary because most of the CRs are ill-structured with inconsistent information. Therefore, student designers must be systemically guided to abstract useful information from CRs.

Recommender system is an important theoretical foundation of the proposed CR-FF method. Recommender systems are broadly used by ecommerce platforms to recommend new product/service (e.g., restaurant movie, book, news, jobs, services, education, etc.) to customers. A number of practically feasible recommendation approaches, techniques, and algorithms have been developed [26]. Recommender system is essentially an information filtering system. The most commonly used recommendation approaches include collaborative 
filtering, content-based filtering, and hybrid filtering [26]. Collaborative filtering recommends new products to a target customer based on the "collaborative preferences" of similar customers [27]. Content-based filtering makes recommendations based on to what extent a product's key features match with a customer's known preferences. Lastly, the hybrid filtering combines the advantages of collaborative filtering and content-based filtering to address some inherent issues of using either approach alone. If the "design language" is used to interpret the three popular recommendations approaches, collaborative filtering relies on customer-related information to make recommendations, while content-based filtering relies on product-related information to make recommendations. In the previous studies of recommender systems, the unique role played by functions in impacting customer's rating for a product has not been extensively studied.

The applications of recommender systems can also be found in the area of technologyenhanced learning [28]. Some learning recommender systems have been developed to recommend personalized learning contents and assignments to students [29-30]. Few efforts have been devoted to developing specialized recommender systems for design practice and education, apart from some notable exceptions. For example, Fuge et al. developed a design recommender system to recommend the most relevant design method to designers based on the textual analysis of problem statement [31]. Liu et al. developed a function recommender system (FRS) to recommend novel functions to an existing product based on the content analysis of online customer reviews [32].

\section{Framework of CR-driven function formulation}

As illustrated in Figure 2, the customer review driven function formulation (CR-FF) method is developed by following the system-of-systems integration strategy, which means the novel integration of separate resources from a collection of sub-systems toward a more complex, functional, and capable new system. Customer reviews (CR) are published by end users on the ecommerce platforms that are built by the online retailers. Therefore, the values of CRs are mostly explored in the customer domain and business domain. Recommender systems are used by retailers to recommend new product/service to customers, therefore, the values of recommender systems are mainly explored in the customer domain and business domain as well. Additionally, there are some previous studies of the applicability of CRs and recommender system for product planning in the factory domain [3].

In this study, customer review, design method, and recommender system will be effectively integrated to support the project-based learning based on engineering design courses. In other words, for the first time, the values of CRs are being explored in the engineering education domain. Similarly, learning factory can be regarded as another application of the system-ofsystems integration strategy for engineering education, in which, various manufacturing systems, technologies, tasks, and data are brought from factory to university campus to support the project-based learning in design and manufacturing [33]. 


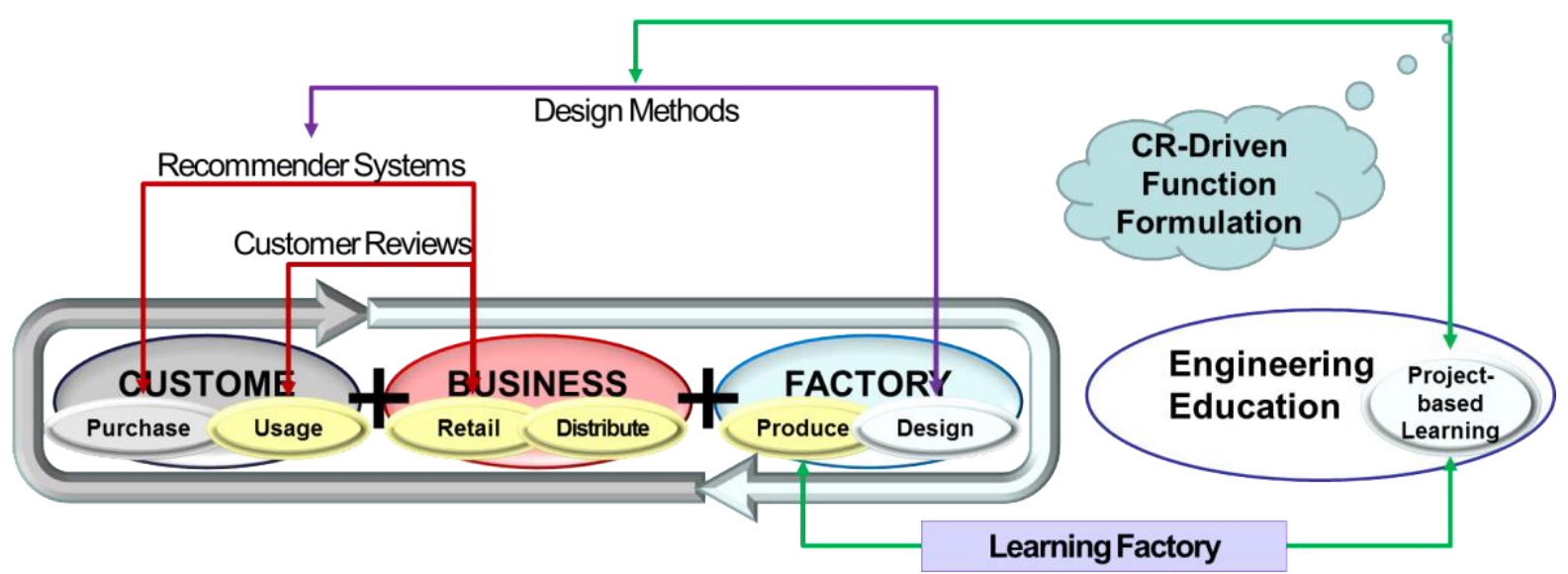

Figure 2. System-of-systems approach for engineering education

Figure 3 illustrates the conceptual framework of the CR-driven function formulation. Firstly, student designers are taught a variety of design methods and a systematic design process (e.g., Functional Basis, Axiomatic Design, Morphological Chat, TRIZ, etc.). Secondly, student designers are taught to analyze the massively crowdsourced customer reviews, through qualitative and quantitative data analysis, to abstract useful design information, knowledge and proposition. Thirdly, student designers are guided to follow the design methods to query, enrich, and restructure the abstracted customer propositions to perform design activities such as problem definition, function formulation, concept generation, and concept improvement.

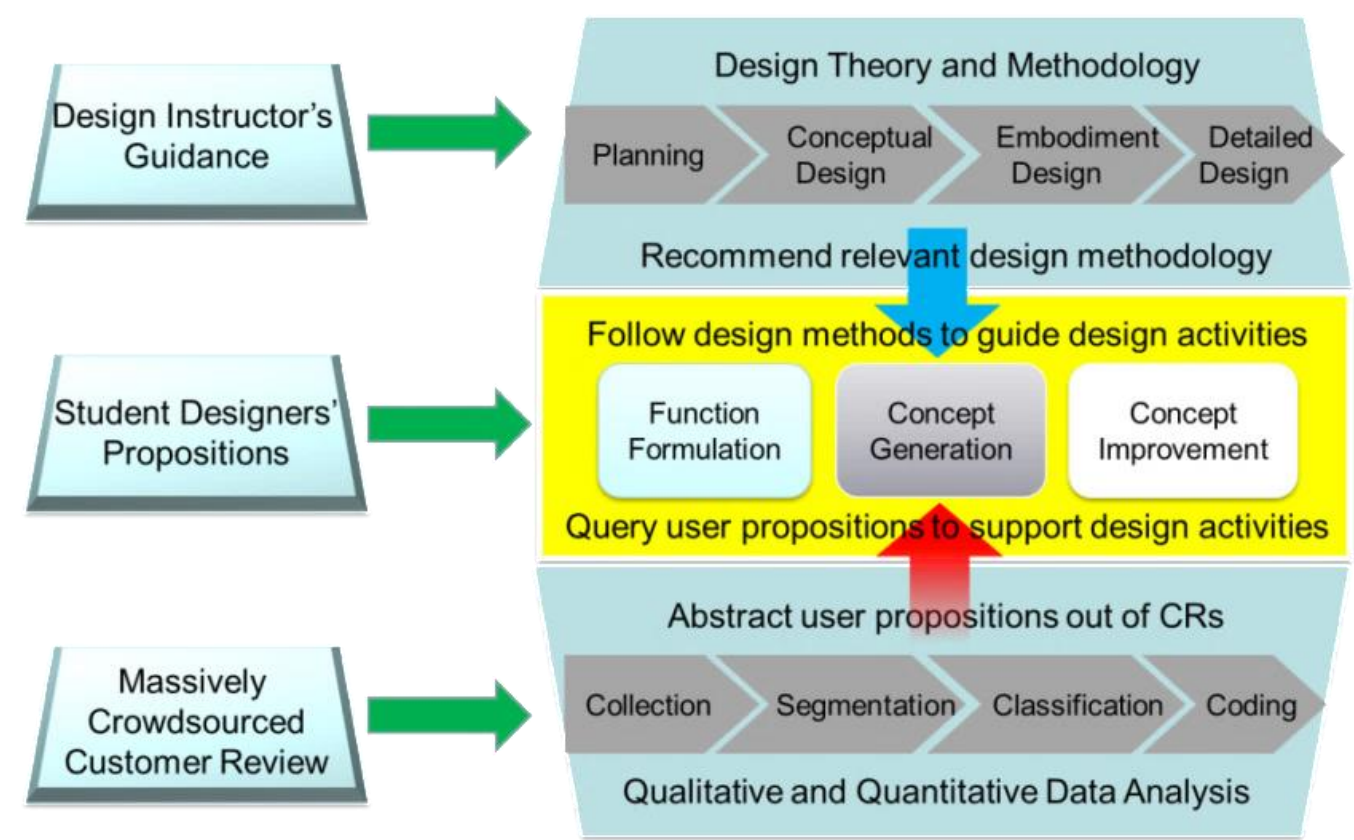

Figure 3. Conceptual framework of CR-driven function formulation

\section{Process of CR-driven function formulation (CR-FF)}

Based on information abstracted from CRs, the proposed CR-FF process guides student designers to estimate an unknown rating that customers would give to a candidate function $f$, which is not carried by the target product $p$ yet, formally $R(p, f)$. Specifically, a candidate function $f$ is abstracted from a peer product $p^{\prime}$ that is similar to the target product $p$, then $R(p, f)$ is estimated based on the similarity of $p^{\prime}$ to $p$ and the rating of $f$ on $p^{\prime}$. The inputs of CR-FF include a target product, multiple CRs about this product, and a portrait of target 
customers (i.e., user demographics such as age, education, occupation, etc.). The output of $\mathrm{CR}-\mathrm{FF}$ is a list of new functions that are recommended for the target product. As illustrated in Figure 4, a systemic CR-FF process consists of 6 steps:

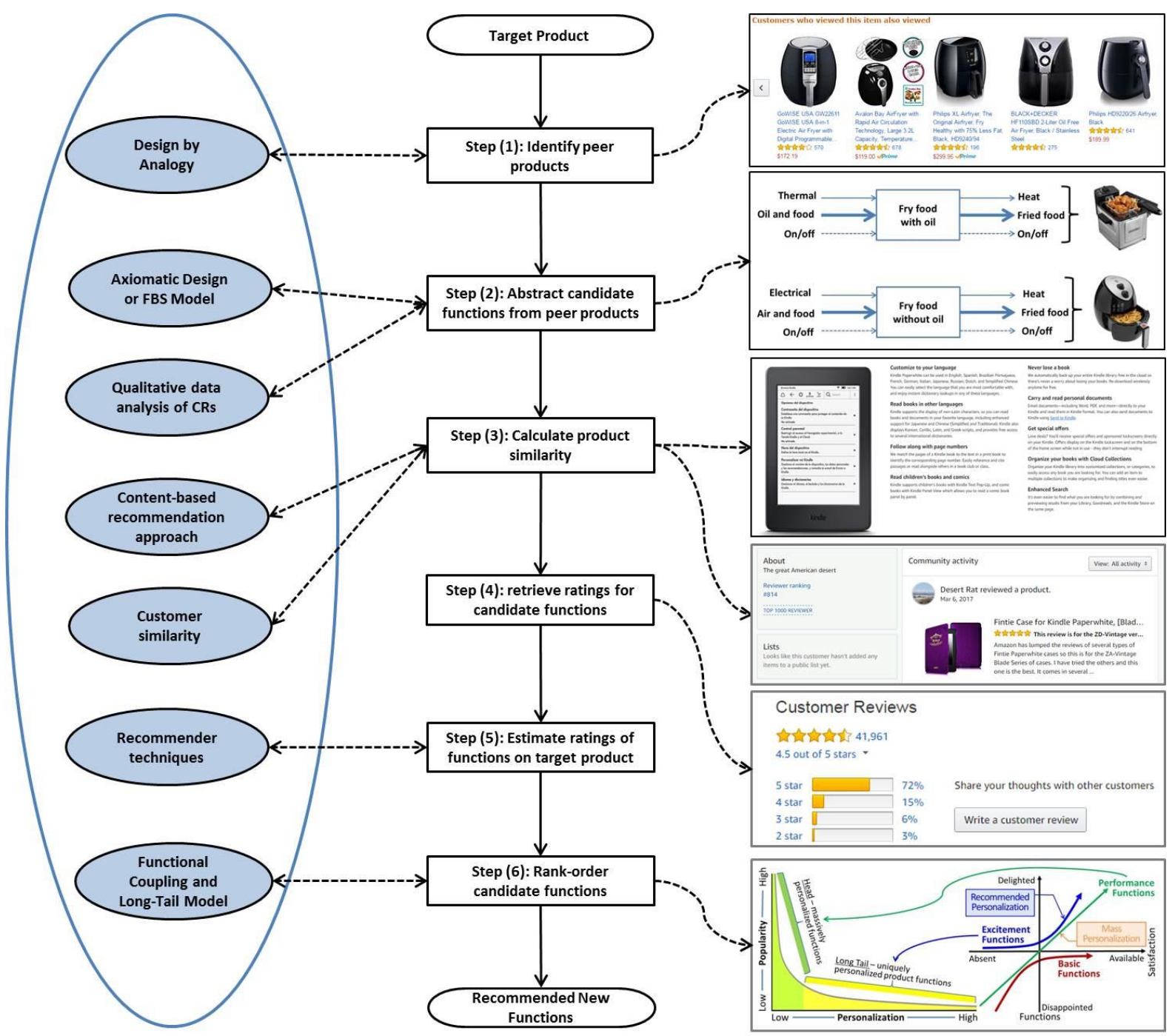

Figure 4. Systematic process of CR-driven function formulation

Step (1): Identify peer products for the target product

Student designers are guided to identify multiple peer products that are related to the target product. Design by analogy and case-based reasoning are important theoretical foundations for this step [34-35]. Designers can follow the design-by-analogy methods (e.g., the wordtree design-by-analogy [36]) to identify peer products. Peer products can be identified based on information regarding customer domain, functional domain, and physical domain. Firstly, peer products can be identified through customer need, behavior, and preference. If two products are intended to fulfill the same customer need, they can be regarded as similar products. If two products are often searched, purchased, and reviewed together by the same customers, they can be regarded as similar products. It should be noted that Amazon.com automatically recommends peer products through "customers who bought this item also bought". Secondly, if two products share some common functions (especially the same set of highly rated functions) before, they can be regarded as similar products. Thirdly, if two 
products share some common components, structures, or materials, they can also be regarded as similar products.

Step (2): Abstract candidate functions from peer products

Those functions carried by the peer functions, which are not carried by the target product, are considered candidate functions that can be potentially recommended. Candidate functions can be abstracted from a peer product through two strategies. Firstly, designers can follow the functional modeling methods to create and then compare the functional chains between a candidate product and its peer products. For example, the zigzagging process of Axiomatic Design can be followed to create a functional hierarchy for every peer product [10]. Then each peer product is compared to the target product, with respect to their functional hierarchies, to make visible the candidate functions.

Secondly, designers can follow a qualitative data analysis process to directly abstract candidate functions based on the content analysis of "product descriptions" and "customer reviews" published on Amazon.com. The former is published by manufacturers, whereas the latter is published by end users. A systematic qualitative data analysis process consists of 5 sub-steps [37]: (a) data collection, (b) data transcription, (c) data segmentation, (d) data categorization, and (e) data coding. After a product description or customer review is segmented into multiple individual coding units (i.e., short phrases), designers can follow a particular design methodology to develop a categorization scheme, and then assign the most suitable code to each coding unit. Depending on the design methodology taught in class, the categorization scheme varies respectively. For example, if the Axiomatic Design Theory was taught, then designers could assign the codes such as CN (customer need), FR (functional requirement), DP (design parameter), and DC (design constraint), as well as the design relationships such as CN-FP, FR-FR, FR-DP, etc. If the Function-Behavior-Structure (FBS) framework is taught, then designers should assign the codes such as $\mathrm{R}$ (requirement), $\mathrm{F}$ (function), Be (expected behavior), Bs (actual behavior), and S (structure), as well as the design operations such as formulation, synthesis, analysis, and evaluation.

The candidate functions abstracted from the peer products can be represented in different formats. Two commonly used representations are "verb+noun" pairs and "input-output transformations" of energy, material, and signal.

Step (3): Calculate similarity between peer product and target product

Student designers are guided to calculate the similarity of a peer product $p^{\prime}$ to the target product $p$, formally $\operatorname{sim}\left(p, p^{\prime}\right)$. Inspired by the content-based recommendation approach, the similarity between two products can be indirectly measured through the similarity between product-related CRs, or $\operatorname{sim}\left(c, c^{\prime}\right)$, based on the measure of term frequency inverse document frequency (TFIDF) [38]. Specifically, $\operatorname{sim}\left(c, c^{\prime}\right)$ is calculated via Equation (1), where $K$ refers to the keywords shared by the CRs of the target and peer product, and $w_{i, p}$ and $w_{i, p}$, mean the weight of keyword $k_{i}$ in the CR of the target and peer product, respectively. There are plenty of online tools that can be used to calculate the similarity between two text-based contents (CRs), therefore, it is unnecessary for student designers to manually calculate $\operatorname{sim}\left(c, c^{\prime}\right)$. 


$$
\operatorname{sim}\left(c, c^{\prime}\right)=\cos \left(\vec{w}_{p}, \vec{w}_{p^{\prime}}\right)=\frac{\vec{w}_{p} \cdot \vec{w}_{p^{\prime}}}{\left\|\vec{w}_{p}\right\|_{2} \times\left\|\vec{w}_{p^{\prime}}\right\|_{2}}=\frac{\sum_{i=1}^{K} w_{i, p} w_{i, p^{\prime}}}{\sqrt{\sum_{i=1}^{K} w_{i, p}^{2}} \sqrt{\sum_{i=1}^{K} w_{i, p^{\prime}}^{2}}}(1)
$$

Since multiple CRs for the same peer product can be used to calculate product similarity, based on the assumption that not every CR has equal value, designers should assign a weighting factor to each $\mathrm{CR}$, or formally $w_{c \text { r }}$. The weight of a CR can be determined through different strategies. Firstly, the weight is related to the similarity between the reviewer and the target customer. In other words, the more similar between reviewer and the target customer, the higher weight this reviewer's CR content should count. Secondly, customer similarity can be estimated based on customers' demographic information (e.g., age, gender, occupation, education), as well as their previous purchase history. The demographic information can be abstracted based on qualitative data analysis of CRs. Thirdly, the weight of a CR can be determined by the reviewer ranking, the number of "people found this CR helpful", and the number of comments left under a CR, which are all public information on Amazon.com.

Product similarity can be calculated by aggregating the content similarity between multiple CRs for the peer product with the most representing CR for the target product, as well as the weighting factors for each CR. The weighted sum approach can be used, via Equation (2)

$$
\operatorname{sim}\left(p, p^{\prime}\right)=\sum_{c^{\prime} \in \hat{C}} w_{c^{\prime}} \times \operatorname{sim}\left(c, c^{\prime}\right)
$$

It should be noted that, most of the CRs contain many distracting "noises" (e.g., spelling errors, nonsensical descriptions, emotional expressions, etc.) that must be filtered to increase the accuracy of $\operatorname{sim}\left(c, c^{\prime}\right)$. Therefore, as much as possible, designers should identify, remove these noises from the selected CRs before using them to calculate product similarity.

Step (4): Retrieve ratings for candidate functions on peer products

Student designers are guided to retrieve the known ratings for candidate functions on the peer product, formally $R\left(p^{\prime}, f\right)$. Most of the ecommerce platforms (e.g., Amazon.com) only allow customers to rate a product as a whole instead of rating its individual functions. Some travel websites allow users to rate different aspects of a hotel such as cleanliness, comfort, location, room service, etc. As a result, the product rating is directly used as the rating for all the candidate functions abstracted from the same peer product. In other words, the underlining assumption is that all the candidate functions abstracted from the same peer product will receive the same rating. Such an assumption is only valid for those simple products that carry a few functions as opposed to the complex products that carry many functions. Alternatively, since CRs often disclose specific reasons why a product is highly rated or otherwise, designers can also perform a qualitative data analysis process to uncover the correlation between individual functions and the overall product rating.

Step (5): Estimate the rating of candidate functions on the target product

Based on the above-calculated product similarity, and ratings of candidate functions on peer products, student designers are guided to estimate the rating of every candidate function on the target product, formally $R(p, f)$. Specifically, $R\left(p^{\prime}, f\right)$ is weighted by the product similarity of $\operatorname{sim}\left(p, p^{\prime}\right)$. In other words, the more similar between a peer product $p^{\prime}$ and the target product $p$, the higher weight $R\left(p^{\prime}, f\right)$ counts toward estimating $R(p, f)$. Let $\hat{P}_{f}$ 
denote a set of peer products that all carry a particular function $f, R(p, f)$ is predicted via Equation (3), where $t$ is a normalizing factor often set to be $t=1 / \max _{p \in \hat{P}} \operatorname{sim}\left(p, p^{\prime}\right)$. If a candidate function $f$ is shared by multiple peer products, the maximum result of $\operatorname{sim}\left(p, p^{\prime}\right) \times$ $R_{u}\left(p^{\prime}, f\right)$ is picked out. If a candidate function is only carried by one peer product, its rating is naturally promoted to be the maximum.

$$
R(p, f)=t \times \max _{p^{\prime} \in \hat{P}_{f}} \operatorname{sim}\left(p, p^{\prime}\right) \times R\left(p^{\prime}, f\right)
$$

Step (6): Rank-order candidate functions

Finally, student designers are guided to rank-order all candidate functions. In addition to the estimated ratings, some other factors should be considered to finalize the ranking. Firstly, according to the Independence Axiom of Axiomatic Design [10], the candidate functions that couple with the existing functions of the target product should be demoted in the ranking. Secondly, based on the Long Tail Theory and the Kano Customer Satisfaction Model [39-40], the priority of recommendations should be given to those unpopular functions that will trigger user excitement. Popularity is a relative concept, which can be measured by counting how many products are carrying the same function. Thirdly, the candidate functions that can be fulfilled by means of the existing components of the target product should be promoted in the ranking. After multiple most recommendable functions are selected, they can be incorporated into the functional hierarchy of the target product according to the dependency relationships, which concludes a CR-driven function formulation process.

\section{Illustrative example}

The CR-FF method was taught based on a second-year engineering design course (i.e., MMAN2100 Engineering Design II) at University of New South Wales in the 2017 fall semester. The course enrolled 408 sophomore/junior undergraduates who major in mechanical engineering, mechatronic engineering, manufacturing engineering, etc. All the students were tasked, in the format of an individual design exercise, to follow the CR-FF process to recommend new functions for a robotic vacuum cleaner. Table 1 summarizes the requirements of this design exercise. The exercise counted $5 \%$ of a student's final grade. Upon the permission of one student, his design result is presented as an illustrative example.

Table 1. Requirements of the design exercise

\begin{tabular}{|c|c|c|}
\hline Target Product & iRobot Roomba 650 Robotic Vacuum Cleaner. & Design Method \\
\hline \multirow{5}{*}{$\begin{array}{l}\text { Task } \\
\text { Specification }\end{array}$} & $\begin{array}{l}\text { (1) Abstract a set of CNs based on the customer } \\
\text { reviews about the target product on Amazon.com }\end{array}$ & $\begin{array}{l}\text { Qualitative Data } \\
\text { Analysis }\end{array}$ \\
\hline & $\begin{array}{l}\text { (2) Identify } 4-5 \text { peer products that are similar or related } \\
\text { to the target product }\end{array}$ & Design by Analogy \\
\hline & $\begin{array}{l}\text { (3) Abstract } 9 \text { candidate functions based on peer } \\
\text { products, and represent them in the form of < verb- } \\
\text { object }>\text { by following the Functional Basis Method }\end{array}$ & Functional Basis \\
\hline & $\begin{array}{l}\text { (4) Classify candidate functions based on the Kano } \\
\text { Model and Long Tail Model }\end{array}$ & Kano Model \\
\hline & $\begin{array}{l}\text { (5) Rank order all the candidate FRs and organize them } \\
\text { into a hierarchy }\end{array}$ & Function Hierarchy \\
\hline
\end{tabular}

Firstly, the student collected a total of $60 \mathrm{CRs}$ from multiple ecommerce platforms such as Amazon.com, Ebay.com, Alibaba.com, and Jingdong.com. The customer needs are summarized in Table 2. 
Table 2. Result of CN abstraction

\begin{tabular}{lc}
\hline \multicolumn{1}{c}{ Customer Need } & Frequency \\
\hline iRobot should clean the dirt effectively & 19 \\
\hline iRobot should manage the cleaning schedule reasonably & 7 \\
\hline iRobot should navigate accurately & 17 \\
\hline iRobot should identify terrains and obstructions accurately & 19 \\
\hline iRobot should be easy to use & 5 \\
\hline iRobot should transverse between different terrains(mainly carpets) effectively & 22 \\
\hline iRobot should have compatibility with smart home controller & 8 \\
\hline iRobot cleaning should cover the whole room especially corners & 11 \\
\hline iRobot should clean quietly & 16 \\
\hline iRobot should have a battery with larger volume & 8 \\
\hline iRobot should charge the battery fast & 4 \\
\hline iRobot should not damage furniture & 3 \\
\hline
\end{tabular}

Next, the student followed three strategies to search for peer products: (1) the products that carry similar functions with the target product, (2) the products that are equipped with similar design parameters with the target product, and (3) the products that are often searched together with the target product. Specifically, "detect rubbish", "clean dirt", and "make schedule" were used as keywords to search for peer products with similar functions; "round shape", "rotating brush", and "digital panel" were used as keywords to search for peer products with similar design parameters. Moreover, the ecommerce platforms would automatically display a list of products that were often purchased together with iRobot. Table 3 summarizes the selection of five peer products. Figure 5 shows a set of candidate functions that were abstracted from these peer products.

Table 3. Selection of peer products

\begin{tabular}{ll}
\hline Peer Products & Identification Criteria \\
\hline Water Spray Mop with 360 Degree & Same Function(Clean Dirt); \\
Spinning Head & Commonly Searched Together \\
\hline Floor Waxing and Polishing Machine & Similar Design Parameters(Round Shape, Rotating Brush) \\
\hline Air Cleaner with Colourful Light & $\begin{array}{l}\text { Similar Design Parameters(Round Shape, Digital Panel); Commonly } \\
\text { Searched Together }\end{array}$ \\
\hline Air Humidifier & $\begin{array}{l}\text { Similar Design Parameters(Round Shape, Digital Panel); Commonly } \\
\text { Searched Together }\end{array}$ \\
\hline Aromatherapy Diffuser with & $\begin{array}{l}\text { Similar Design Parameters(Round Shape, Digital Panel); Commonly } \\
\text { Colourful Light }\end{array}$ \\
\hline
\end{tabular}




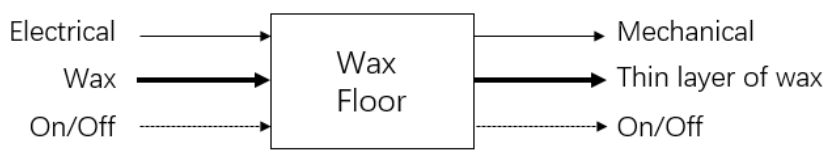

Function is carried by floor waxing and polishing machine

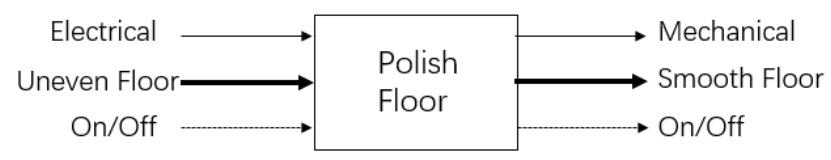

Function is carried by floor waxing and polishing machine

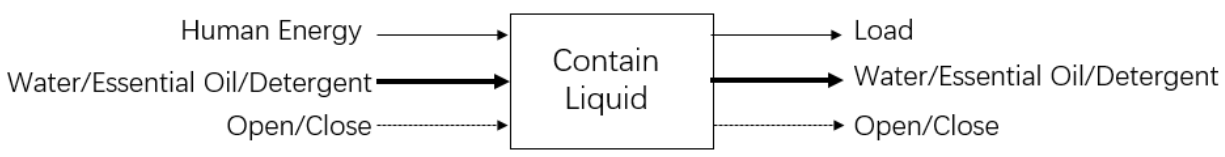

Function is carried by water spray mop, air humidifier, aromatherapy diffuser
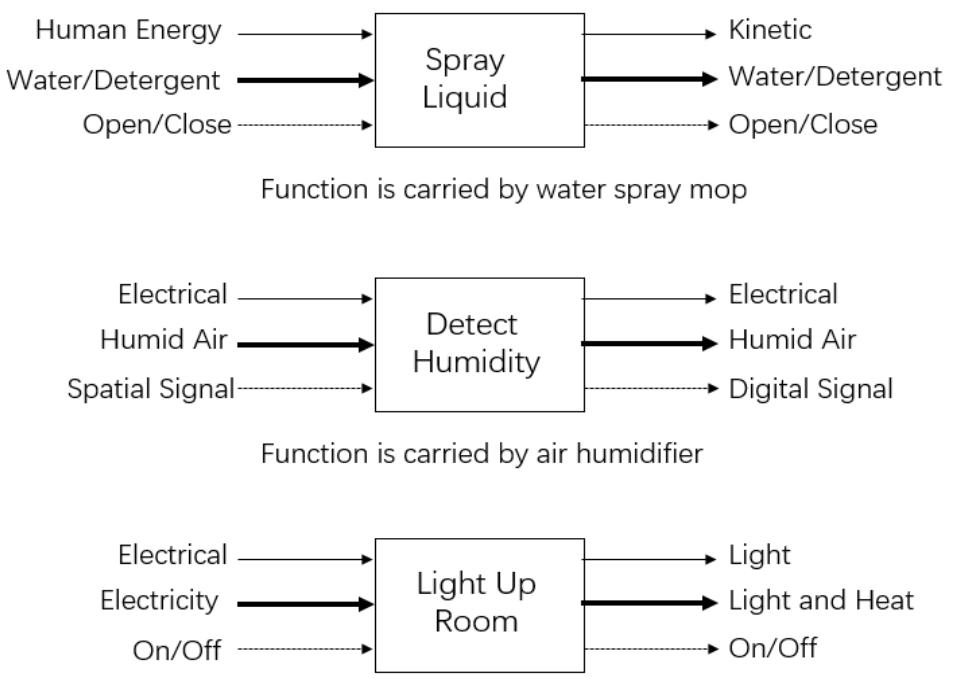

Function is carried by air diffuser and cleaner(with colorful light)

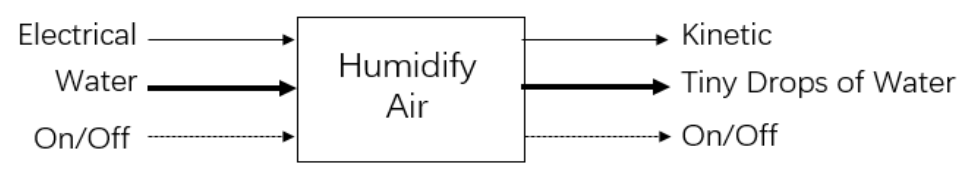

Function is carried by air humidifier

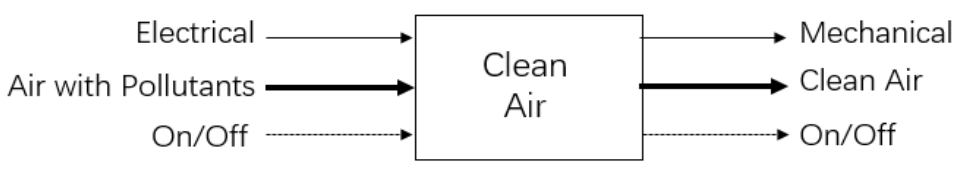

Function is carried by air cleaner

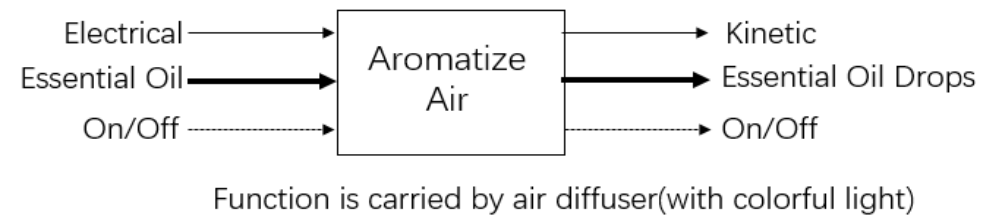

Figure 5: Candidate functions abstracted from peer products 
Next, in consideration of the customer needs abstracted from CRs, the student followed the Kano Customer Model to classify candidate functions into three categories: basic function $(\mathrm{BF})$, performance function (PF), and excitement function (EF). Table 4 shows the classification result and rationale.

Table 4. Classification of candidate functions

\begin{tabular}{lll}
\hline FRs & Category & Classification Reason \\
\hline Wax Floor & EF & No comments expected iRobot to wax \\
\hline Polish Floor & PF & $\begin{array}{l}\text { Some customers thought iRobot could iron the carpet, they might require } \\
\text { iRobot to polish floor sooner }\end{array}$ \\
\hline Contain Water & BF & $\begin{array}{l}\text { Some customers were disappointed that iRobot could not mop floor while } \\
\text { Ecovacs could. }\end{array}$ \\
\hline $\begin{array}{l}\text { Detect } \\
\text { Humidity }\end{array}$ & EF & This FR was just commented under humidifier and air-conditioner \\
\hline Spray Water & BF & The same as 'Contain Water' \\
\hline Light Up Room & EF & No comments mentioned it \\
\hline Humidify Air & PF & $\begin{array}{l}\text { Some customers showed expectation that iRobot should be able to } \\
\text { humidify air but the tone was not strong. }\end{array}$ \\
\hline Clean Air & EF & Customers currently just require it to clean the floor \\
\hline Aromatize & EF & This FR was just commented under aromatherapy diffuser \\
\hline
\end{tabular}

Finally, the student ranked the candidate functions against their applicability for the target product. In other words, the easier a function could be transferred to the target product, the higher it was ranked. Table 5 summarizes the final ranking of candidate functions.

Table 5. Ranking of candidate functions

\begin{tabular}{cl}
\hline Ranking & FRs \\
\hline 1 & Contain Water \\
\hline 2 & Clean Air \\
\hline 3 & Wax Floor \\
\hline 4 & Spray Water \\
\hline 5 & Aromatize Air \\
\hline 6 & Detect Humidity \\
\hline 7 & Humidify Air \\
\hline 8 & Polish Floor \\
\hline 9 & Light Up Room \\
\hline
\end{tabular}

\section{Conclusion and future work}

This study illuminates the unique values of massively crowdsourced customer reviews (CRs) for engineering design education. A new CR-driven function formulation method is prescribed. The method is characterized by a novel integration of design methods (i.e., Axiomatic Design, FBS ontology, design by analogy, functional modeling, etc.), recommendation techniques (i.e., content analysis, customer similarity, and rating prediction), and information contained in customer reviews (i.e., product description, product rating, and reviewer ranking). Student designers can follow the proposed new method to abstract useful information from the massively crowdsourced CRs to support their project-based learning in product design.

The proposed method has some limitations. Firstly, sometimes the content of CRs contains many "noises" (e.g., complaint about product delivery and customer service) that are irrelevant to design, while it is too time-consuming to conduct a thorough qualitative data 
analysis to completely remove all the noises. Secondly, from time to time, customers do not mean they say in customer reviews. Finally, sometimes the meaning of certain texts will be changed when they are taken out of the particular context. In that regard, a possible solution is to combine the studies of contextual inquiry and context-aware recommendation systems to analyze those context-related design attributes.

With respect to future work, firstly, a controlled design experiment will be conducted to thoroughly validate the effectiveness of the proposed framework in a rigorous manner. Secondly, a mobile tool (i.e., smartphone application) is being developed to facilitate designers to carry out the CR-driven function formulation process by automating some key steps (i.e., calculation of customer similarity and product rating) that involve computing.

\section{Reference}

[1] Li, J., Tao, F., Cheng, Y., \& Zhao, L. (2015). Big data in product lifecycle management. The International Journal of Advanced Manufacturing Technology, 81(14), 667-684.

[2] Jin, J., Liu, Y., Ji, P., \& Liu, H. (2016). Understanding big consumer opinion data for market-driven product design. International Journal of Production Research, 54(10), 3019-3041.

[3] Liu, A., \& Lu, S. C. Y. (2016). A crowdsourcing design framework for concept generation. CIRP Annals-Manufacturing Technology, 65(1), 177-180.

[4] Liu, Y. C., Chakrabarti, A., \& Bligh, T. (2003). Towards an 'ideal'approach for concept generation. Design Studies, 24(4), 341-355.

[5] Jonson, B. (2005). Design ideation: the conceptual sketch in the digital age. Design studies, 26(6), 613-624.

[6] Kruger, C., \& Cross, N. (2006). Solution driven versus problem driven design: strategies and outcomes. Design Studies, 27(5), 527-548.

[7] Nikolaus, F., Von Hippel, E., and Schreier, M. "Finding commercially attractive user innovations: A test of lead-user theory." Journal of product innovation management, 2006, 23(4): 301-315.

[8] Sutton, R.I., and Hargadon, A. "Brainstorming groups in context: Effectiveness in a product design firm." Administrative Science Quarterly, 1996, 41(4): 685-718.

[9] Christina, W. "Ethnography in the field of design." Human organization, 2000, 59(4): 377-388.

[10] Suh, N. P. (2001). Axiomatic Design: Advances and Applications (The Oxford Series on Advanced Manufacturing).

[11] Park, G. J. (2014). Teaching conceptual design using axiomatic design to engineering students and practitioners. Journal of Mechanical Science and Technology, 28(3), 989.

[12] Mustafa Suphi, E., Komoto, H., Van Beek, T.J., D'Amelio, V., Echavarria, E., and Tomiyama, T. "A review of function modeling: Approaches and applications." Artificial Intelligence for Engineering Design, Analysis and Manufacturing, 2008, 22(2): 147-169.

[13] Chen, Y., Zhang, Z.N., Huang, J., and Xie, Y.B. Toward a scientific ontology based concept of function. Artificial Intelligence for Engineering Design, Analysis and Manufacturing, 2013, 27(3): 241-248

[14] Kannengiesser, U., and Gero, J.S. "The situated function-behavior-structure framework". Design Studies, 2004, 25(4): 373-391.

[15] Lu, S.C.Y, and Liu, A. "Subjectivity and objectivity in design decisions." CIRP AnnalsManufacturing Technology, 2011, 60(1): 161-164. 
[16] Eckert, C., Alink, T., Ruckpaul, A., and Albers, A., "Different Notions of Function: Results From an Experiment on the Analysis of an Existing Product". Journal of Engineering Design, 2011, 22(11-12): 811-837.

[17] Vermaas, P.E. "The coexistence of engineering meanings of function: four responses and their methodological implications". Artificial Intelligence for Engineering Design, Analysis and Manufacturing, 2013, 27: 191-202.

[18] Estellés-Arolas, E., \& González-Ladrón-De-Guevara, F. (2012). Towards an integrated crowdsourcing definition. Journal of Information science, 38(2), 189-200.

[19] Chatzimilioudis, G., Konstantinidis, A., Laoudias, C., \& Zeinalipour-Yazti, D. (2012). Crowdsourcing with smartphones. IEEE Internet Computing, 16(5), 36-44.

[20] Brabham, D. C. (2008). Crowdsourcing as a model for problem solving: An introduction and cases. Convergence, 14(1), 75-90.

[21] Hills, T. T. (2015). Crowdsourcing content creation in the classroom. Journal of Computing in Higher Education, 27(1), 47-67.

[22] Mudambi, S. M., \& Schuff, D. (2010). What makes a helpful review? A study of customer reviews on Amazon. com.

[23] Hennig-Thurau, T., Malthouse, E. C., Friege, C., Gensler, S., Lobschat, L., Rangaswamy, A., \& Skiera, B. (2010). The impact of new media on customer relationships. Journal of service research, 13(3), 311-330.

[24] Kozinets, R. V. (2002). The field behind the screen: Using netnography for marketing research in online communities. Journal of marketing research, 39(1), 61-72.

[25] Hu, M., \& Liu, B. (2004, August). Mining and summarizing customer reviews. In Proceedings of the tenth ACM SIGKDD international conference on Knowledge discovery and data mining (pp. 168-177). ACM.

[26] Gediminas, A., and Tuzhilin, A. "Toward the next generation of recommender systems: A survey of the state-of-the-art and possible extensions." IEEE transactions on knowledge and data engineering, 2005,17(6): 734-749.

[27] Schafer, J.B., Frankowski, D., Herlocker, J., and Sen, S. "Collaborative filtering recommender systems." In The adaptive web, pp. 291-324. Springer Berlin Heidelberg, 2007.

[28] Manouselis, N., Drachsler, H., Vuorikari, R., Hummel, H., \& Koper, R. (2011). Recommender systems in technology enhanced learning. In Recommender systems handbook (pp. 387-415). Springer US.

[29] Hsu, M. H. (2008). A personalized English learning recommender system for ESL students. Expert Systems with Applications, 34(1), 683-688.

[30] Bobadilla, J. E. S. U. S., Serradilla, F., \& Hernando, A. (2009). Collaborative filtering adapted to recommender systems of e-learning. Knowledge-Based Systems, 22(4), 261265.

[31] Fuge, Mark, Bud Peters, and Alice Agogino. "Machine Learning Algorithms for Recommending Design Methods." ASME Trans. on Journal of Mechanical Design, 2014,136(10): 101103.

[32] Liu, A., Lu, S., Zhang, Z., Li, T., \& Xie, Y. (2017). Function recommender system for product planning and design. CIRP Annals-Manufacturing Technology.

[33] Lamancusa, J. S., \& Simpson, T. W. (2004, October). The learning factory-10 years of impact at penn state. In International Conference on Engineering Education (pp. 16-21).

[34] McAdams, D.A., and Wood, K.L. "A quantitative similarity metric for design-byanalogy." Transactions-ASME Journal of Mechanical Design, 2007, 124(2): 173-182.

[35] Fu, K., Chan, J., Cagan, J., Kotovsky, K., Schunn, C., and Wood, K.L. "The meaning of "near" and "far": the impact of structuring design databases and the effect of distance of analogy on design output." Journal of Mechanical Design, 2013, 135(2): 021007. 
[36] Linsey, J. S., Markman, A. B., \& Wood, K. L. (2012). Design by analogy: A study of the WordTree method for problem re-representation. Journal of Mechanical Design, 134(4), 041009.

[37] Richards, L. (2014). Handling qualitative data: A practical guide. Sage.

[38] Yang, M.C., Wood, W.H., and Cutkosky, M.R. "Design information retrieval: a thesauribased approach for reuse of informal design information." Engineering with Computers, 2005, 21(2): 177-192.

[39] Anderson, C. (2006). The long tail: Why the future of business is selling less of more. Hachette Books.

[40] Sauerwein, E., Bailom, F., Matzler, K., \& Hinterhuber, H. H. (1996, February). The Kano model: How to delight your customers. In International Working Seminar on Production Economics (Vol. 1, No. 4, pp. 313-327). Innsbruck. 